Results: The results showed a significant $(\mathrm{P}<0.024)$ irregularity of oestrous cycle and reduction in relative ovarian weights of experimental animals treated by FSA extract in comparison to the control group. A reduction in the number of secondary follicles and corpora lutea was found in the treated groups as well as an increment in the number of antral follicles in all treated groups when compared with the control.

Conclusion: The observation of irregular oestrous cycle, significant decrease in the number of secondary and antral follicles, and significant increase in the number of atretic follicles demonstrated the potential anti-fertility effects of fenugreek seeds on the female rats were found in this study.

Clinical

\title{
SUBDERMAL ETONOGESTREL: THE EFFECTS ON MENSES, GENERAL HEALTH, RELIGIOUS OBLIGATIONS AND SEXUAL RELATIONSHIP
}

S I Sharifah $A^{1}$, F Dalia Abdulwahab ${ }^{2}$ and A Mokhtar MD ${ }^{2}$

${ }^{1}$ Department of Obstetrics and Gynaecology, Hospital Tengku Ampuan Afzan, Kuantan, Pahang. ${ }^{2}$ Department of Obstetrics and Gynaecology, International Islamic University Kuantan,Pahang

Objective: The aim of this study is to determine the effects of subdermal etonogestrel implant on menstrual pattern, body mass index (BMI), systolic and diastolic blood pressure (SBP and DBP), sexual relationship and religious obligations.

Methodology: A descriptive study was conducted at a family planning clinic between1st January 2010 to 31st December 2011 whereby all the women who had their subdermal etonogestrel removed during this period were included. A selfprepared questionnaire was filled up with the help of a research assistant, which looked into the menstrual cycles prior to and during the implant usage, the non-menstrual adverse events, effect of menses on religious obligations and sexual relationship and the overall acceptability of subdermal etonogestrel as a contraceptive method. Blood pressure (BP) and BMI were recorded at the time of removal, while BP and BMI at insertion were gathered from the case file.

Result: 50 patients were recruited in this study. The mean duration of use was 33.8 months $( \pm 10.4)$ and $78 \%$ had completed 3 years. The prevalence of menstrual disturbance whilst on subdermal etonogestrel implant was $78 \%$. The mean differences of SBP, DBP and BMI at removal and prior to usage were statistically significant. There was no association between BMI and menstrual pattern whilst on implant. Practicing religious obligations and sexual activity were affected in $14.0 \%$ and $18.0 \%$ respectively. Overall satisfaction of subdermal implant was $92 \%$.

Conclusion: Despite the high prevalence of menstrual disturbance, subdermal etonogestrel is well accepted. Changes in the mean of SBP, DBP and BMI were not clinically significant, but a significant proportion of the studied women had a significant increase in SBP, DBP and BMI. The performing of religious duty and sexual activity seem to be unaffected.

\section{LETHAL FETAL ANOMALIES: ARE WE ON THE RIGHT DIRECTION? \\ Dalia F Abdulwahab ${ }^{1}$, Hamizah Ismail ${ }^{1}$, Zalina Nusee ${ }^{1}$, Yong S.Leong ${ }^{2}$ and A Mokhtar MD ${ }^{1}$ \\ ${ }^{1}$ Department of Obstetrics and Gynaecology, International Islamic University Malaysia, Kuantan, Pahang, Malaysia. \\ ${ }^{2}$ Department of Obstetrics and Gynaecology, Hospital Tengku Ampuan Afzan, Kuantan, Pahang, Malaysia}

Objectives: To review the gestational age at diagnosis, method of diagnosis, pregnancy outcome and maternal complications of prenatally diagnosed lethal foetal anomalies.

Methodology: A one year retrospective review of 25 women who had aborted or delivered foetuses with lethal anomalies in a tertiary hospital in 2011 based on patient medical records. 